\title{
Pressure-induced Structures and Structural Evolution in Iron
}

\author{
Li Qi ${ }^{a}$, Shidong Feng ${ }^{a}, \mathrm{Na} \mathrm{Xu}^{a}$, Mingzhen Ma ${ }^{a}$, Qin Jing ${ }^{a}$, Gong Li ${ }^{a}$, Riping Liu ${ }^{a *}$ \\ ${ }^{a}$ State Key Laboratory of Metastable Materials Science and Technology, \\ Yanshan University, Qinhuangdao 066004, PR China
}

Received: September 10, 2014; Revised: August 24, 2015

\begin{abstract}
Molecular-dynamic simulations have been used to study the structure evolution in iron melts rapidly cooled under different pressures. An extreme cooling rate $\left(4 \times 10^{12} \mathrm{~K} / \mathrm{s}\right)$ was adopted in the cooling process. The simulation results show that at the ambient pressure, martensitic transformation happened. However, at a pressure of $1.4 \mathrm{GPa}$, the system passes from $b c c$ structure to a less closed packed structure which is composed of both full icosahedra clusters and $b c c$ structure. With the increase of pressures, an amorphous state was observed, and a compacted local structure with more defected icosahedra is obtained. This work contributes to a better understanding about the dynamics of phase transitions in iron under high pressure, especially during the extremely fast cooling process.
\end{abstract}

Keywords: molecular-dynamics, pressure, iron, structure

\section{Introduction}

The study of high pressure-induced structure transitions has received much attention ${ }^{1}$. Just like temperature or chemical composition for condensed phases, pressure is becoming an important variable processing parameter. High pressure (HP), which can cause a larger change of atom spacing and chemical bonding, has been found to be a powerful tool for affecting and controlling the nucleation and growth in metallic glasses (MGs). Although some works reveal that the employment of HP promotes solidification, no consensus is reached for understanding of the local structure under $\mathrm{HP}^{[2]}$.

The properties of iron under a high pressure are of great interest because it is a classic transition element and is a dominant component in terrestrial planet cores. At present, many studies have revealed information about the state of crystalline iron and provided insight into the nature of planetary cores. For example, experiments and theoretical calculation suggested that iron present in the Earth's core is stable in a body-centered-cubic phase ${ }^{3-6}$. Lee \& Ray $^{7}$ used molecular dynamic simulation to determine how the particles move relatively to one another during its pressure-induced structural transitions. Theoretical calculations and experiments have been made in the past few years aiming to explain these phase transitions under a high pressure ${ }^{8-10}$. However, the behavior of iron both under a high pressure and at an extremely high cooling rate has not been extensively investigated $^{11}$. In the present study, the transition of iron from the super-cooled liquid to solid under HP is simulated. The classical molecular-dynamic (MD) simulation was used in the isobaric-isoenthalpic (NPT) ensemble which permits us to observe structural changes in the material. The calculations were performed based on the MD simulations with the Sutten-Chen model ${ }^{12}$. In this paper, we made a preliminary analysis about the crystallization behavior of the system. Then the microscopic motions of the particles during the

*e-mail: qili@ysu.edu.cn pressure-induced transition were studied. On these systems, transformations can be obtained by changing experimental conditions such as pressure and temperature.

\section{Simulation Methods}

The realism of a classical MD simulation is dependent on the model chosen considering the conditions under which the simulation is being run. The Sutten-Chen model, a realistic embedded-atom (EAM) potential ${ }^{13}$ modified Johnson potential for $\mathrm{Fe}^{[14]}, \Phi_{M J}\left(r_{I J}\right)$ belonging to a broad class of potentials ${ }^{15}$ is used in this paper. Following Baskes, the total energy is written as

$E_{i}=\frac{1}{2} \sum_{j=1, j \neq i}^{N} \Phi\left(r_{i j}\right)+F\left(\rho_{i}\right)$

The embedding energy has the form

$\overline{\rho_{i}}=\frac{1}{\rho} \sum_{j \neq i} \rho\left(r_{i j}\right)$

Where $\Phi\left(\mathrm{r}_{\mathrm{ij}}\right)$ is a two-body central potential between atom $i$ and $j$ with the separation distance $\mathrm{r}_{\mathrm{ij}}, \mathrm{F}(\rho \mathrm{i})$ is the embedding energy of atom $i$ with the electron density $\rho i$ due to all its neighbors:

$\rho\left(r_{i j}\right)=\exp \left[\beta\left(r / r_{0}-1\right)\right]$

$\beta$ describes the decay of the electron density $F_{0}, \rho_{0}$ and $r_{0}$ are chosen such that the equilibrium energy and lattice spacing do not change as the many-body interaction is introduced.

In this work, based on the NPT, the simulations are performed with the system consisting of several thousands atoms in a cubic box with periodic boundary conditions applied, meaning that if a particle leaves the simulation box, an identical particle enters on the other side. For each quenching experiment, the initial liquid state is obtained by holding the system at $5000 \mathrm{~K}$. The Newton equations were 
integrated using the Verlet ${ }^{16}$ algorithm with a time step of $5 \mathrm{fs}$. The system is melted, homogenized, then quenched by rescaling particle velocities to the room temperature at the given rates $\left(4 \times 10^{12} \mathrm{~K} / \mathrm{s}\right)$ under different pressure (from 0 to $12 \mathrm{GPa}$ ), the pressure is controlled by the Andersen method ${ }^{17}$. It is a method that maintains an external pressure by imposing an artificial force on every atom. This has the advantage of not creating a localized interface between the simulated body and the external pressure source. The dependence of the local structure on the temperature is examined. The pair distribution function (PDF) was computed for different pressure, and the pairs analysis $(\mathrm{PA})^{[18]}$ method is used to identify the glass-forming ability and the structure evolution of the system.

\section{Results and Discussion}

The PDF $g(r)$ is a convenient quantity for examining the atomic configuration. The PDF gives the probability of finding a particle in the distance $r$ from another particle. If we count the appearance of two molecules at separation $r$, from $r=0$ to $r=\infty$, we can get the pair distribution function:

$$
g(r)=\frac{N_{n}(r) V_{n}}{4 \pi r^{2} d r N}
$$

The PDF is a useful tool to describe the structure of a system, particularly of liquids. In a solid, the PDF has an infinite number of sharp peaks whose separations and heights are characteristic of the lattice structure. The PDF of a liquid is intermediate between the solid and the gas, with a small number of peaks as short distances, superimposed on a steady decay to a constant value at longer distances.

Since the PDF pattern is different among liquids, glasses, and crystals, it can be used to differentiate the type of transitions in the metallic melts being rapidly cooled, and to show the temperature range of the transitions. The position and intensity of the peaks can further provide information on the local structure of the metallic glass.

Figure 1 demonstrates the RDF patterns of the system at $1450 \mathrm{~K}$, which is formed at $4 \times 10^{12} \mathrm{~K} / \mathrm{s}$ and under
$0,4,8$ and $12 \mathrm{GPa}$ respectively. At the pressure of $0 \mathrm{GPa}$, the second peak of the RDF is broad and smooth, indicating the melting state of system. At the pressure of 4, 8 and $12 \mathrm{GPa}$, the principal structural features of an amorphous solid are clearly seen. The splitting of the second peak of the RDF pattern is a characteristic feature of amorphous phase formation. For rapidly quenched metals, the splitting of the second peak into two sub-peaks is due to the presence of icosahedron-type clusters. Obviously, the difference between the terminal states of both the systems is related to HP.

The melt of iron transforms into an amorphous state during the fast cooling process under HP. However, how the system transform under relatively low pressure, such as $1 \sim 2 \mathrm{GPa}$ ? It is interesting that when the pressure decreases to $1.4 \mathrm{GPa}$, the unusual RDF pattern has been formed, as shown in Figure 2a. For compare easily, the $f c c$ and $b c c$ RDFs of iron were also displayed (Figure 2b). It can be clearly seen that, at $1.4 \mathrm{GPa}$, the feature of RDF is neither that of $b c c$ nor that of $f c c$. The feature is between the melt state and crystal structure. Some dissimilarity can be clearly observed, especially in the intensity and the position of the peaks of the RDFs. Comparing with the melt state, the peak height

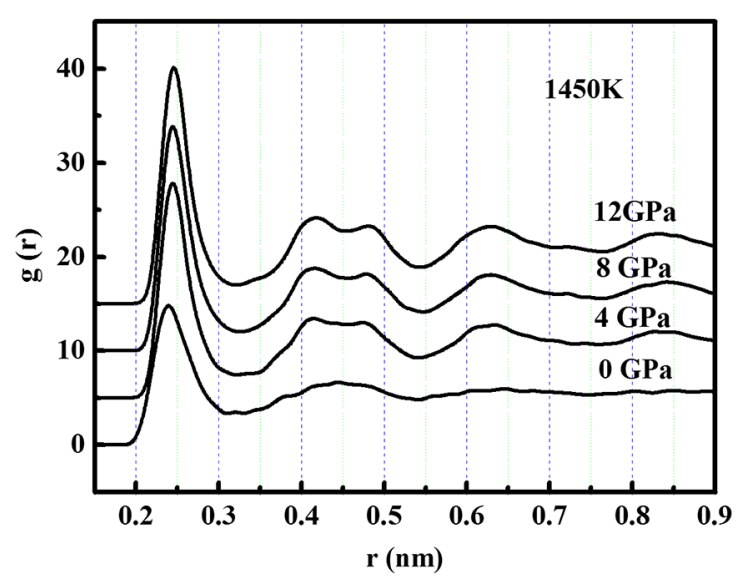

Figure 1. The PDF of iron under different pressures at 1450K.

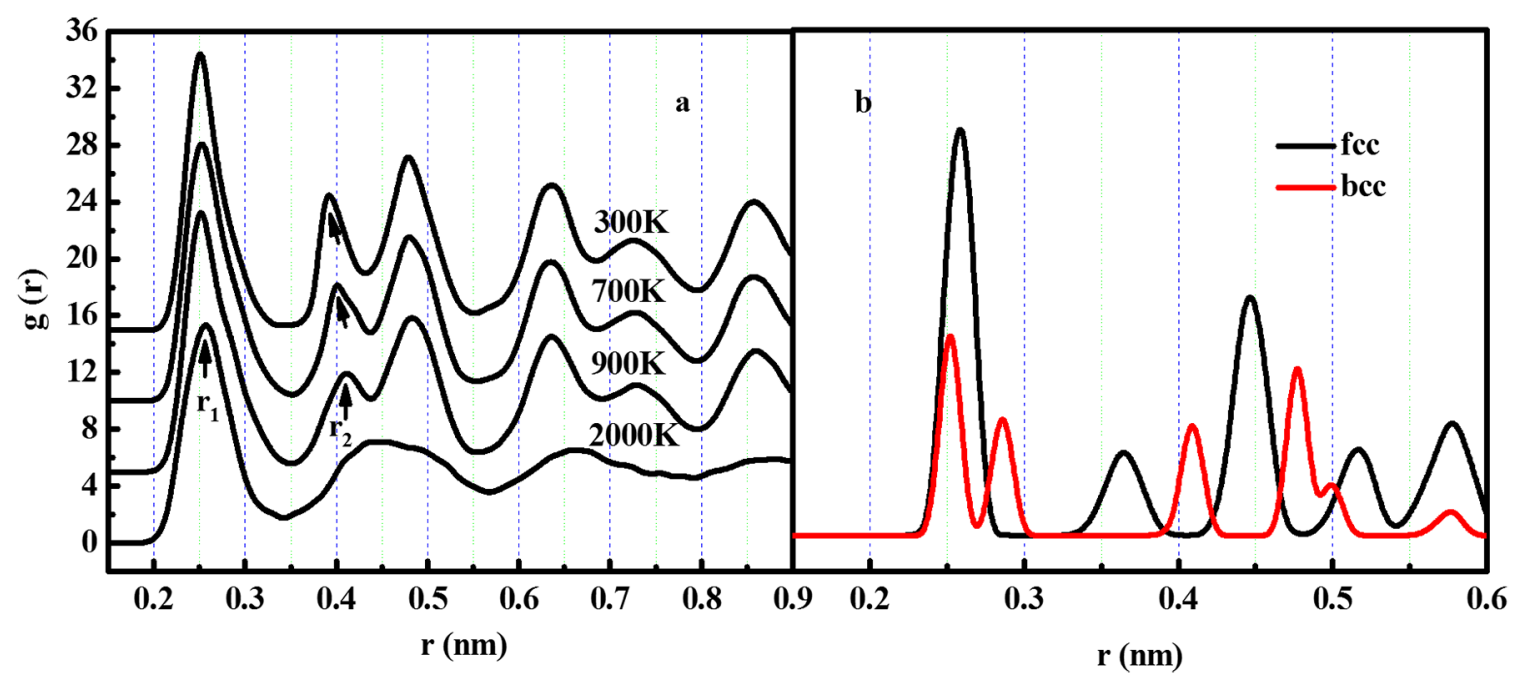

Figure 2. The PDF of iron at different temperatures. left: at 1.4GPa. right: the PDF of fcc and bec of iron. 
and position of the second peak of the RDFs indicate more ordered structure formed in the system. For Fe, the second peak of $b c c$ and $f c c$ is at about $2.8 \AA$ and $3.6 \AA$, respectively. While, in the simulation, the second peak of RDF is at about $4.1 \AA$, which indicates the relative low degree of ordering than crystal structure. Furthermore, both of the intensities of the first two peaks are increased. The position of the first peak, $r_{1}$, remains almost unchanged, but the position of the second peak, $r_{2}$, becomes smaller. This trend indicates that, for the second cell, the number of the atoms rises and the degree of order increases.

The structure evolution of iron from the liquid state with disordered structure to the final states is observed more directly by drawing the projections of all atoms in the system. It should be noted that a clearer visualization would be obtained in a three-dimensional view with crystal rotations. In fact, some differences in terms of homogeneity can already be clearly seen in these two-dimensional cross-sectional projections. Figure 3 shows the projection of all atoms at the cooling rate of $4 \times 10^{12} \mathrm{~K} / \mathrm{s}$ under different pressures, where the volume of the system is normalized in order to make the comparison easier. The figure is the $\mathrm{Y} / \mathrm{X}$ orientation projections of the system, every empty circle represents an iron atom.

Figure $3 \mathrm{a}$ shows the system at room temperature under 4GPa. The system is undoubtedly homogenized and disordered, indicating that the melt state is kept until room temperature and amorphous state is formed which correspond to the RDF of the system (Figure 1). Figures 3b, 3c and 3d show the projections under $1.4 \mathrm{GPa}$ at $1100 \mathrm{~K}, 900 \mathrm{~K}, 300 \mathrm{~K}$, respectively. At pressure of $1.4 \mathrm{GPa}$, the locally ordered structure appears as the temperature decreases to $1100 \mathrm{~K}$ (Figure $3 \mathrm{~b}$ ), and the regular structure enhance evidently at 900K (Figure 3c) which corresponds to the high peak height and wide peak width of the RDF (Figure 2). The lower the temperature is, the higher the degree of ordering of the structure is. Figures $3 \mathrm{e}$ and $3 \mathrm{f}$ shows the projections of $b c c$ and $f c c$ for comparison.

To further understand the effect of the pressure on the local structure evolution and its contribution to the formation of the unusual phase, we examine the local structural information of the simulated systems from the atomic bonding view by means of the Honeycutt-Anderson pairanalysis technique. Some typical bonding pairs are examined, among which 1551 atomic pairs are a characteristic of an icosahedral structure, 1441 pairs are a characteristic of a $b c c$ crystalline structure, 1541 and 1431 pairs are relatively numerous in supercooled liquids and glasses. Figure 4 shows the corresponding structures of these pairs. In comparison with 1551, the other three have some bonds (Atoms that are near-neighbors at each other, as determined by the specified cutoff distance, are connected by "bonds") broken or unformed, forming imperfect structures. One icosahedron (13 atoms with one at center) consists of 121551 pairs. The top and the center atoms in an icosahedron together with their shared five neighboring atoms might form a local structure with 1551 pairs. If one outer bond is broken in

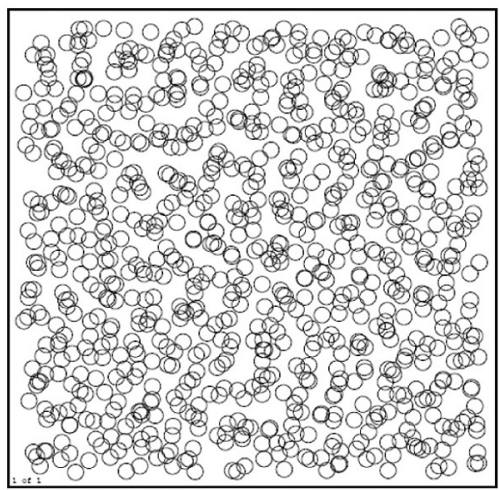

(a)

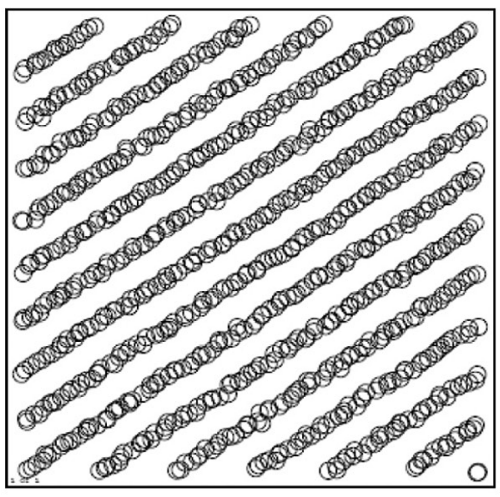

(d)

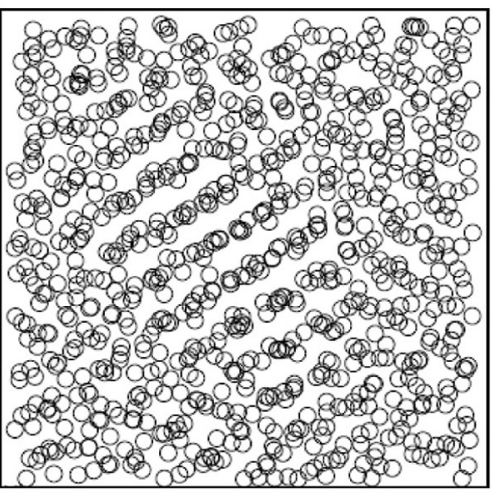

(b)

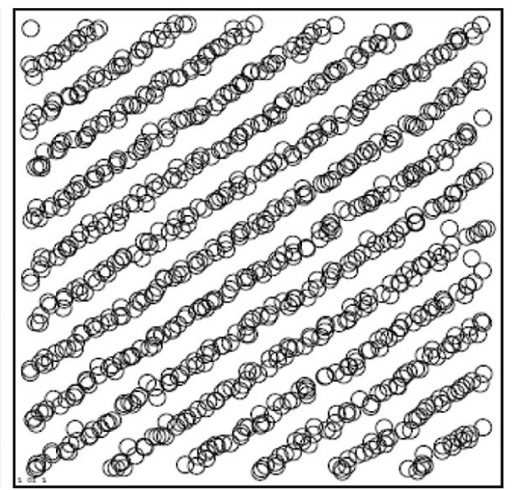

(c) (e)

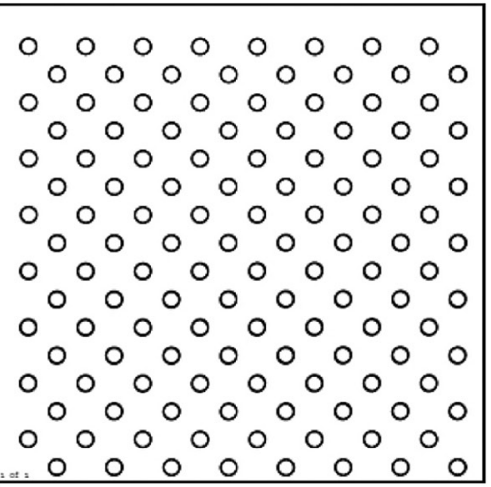

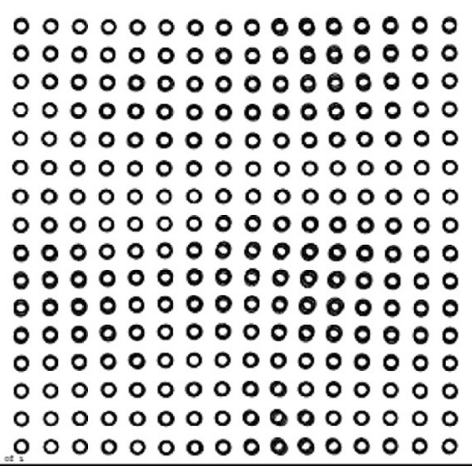

(f)

Figure 3. Two-dimensional cross-sectional projections of the structures of $\mathrm{Fe}$ obtained under different pressures at cooling rate of $4 \times 1012 \mathrm{~K} / \mathrm{s}$. (a) $4 \mathrm{GPa}, 300 \mathrm{~K}$; (b) $1.4 \mathrm{GPa}, 1100 \mathrm{~K}$; (c) $1.4 \mathrm{GPa}, 900 \mathrm{~K}$; (d) $1.4 \mathrm{GPa}, 300 \mathrm{~K}$; (e) bec structure; (f) fcc structure. 

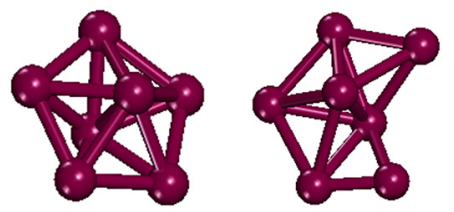

1551

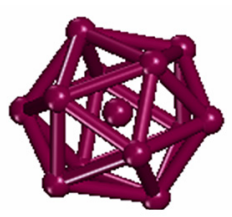

ideal icosahedra

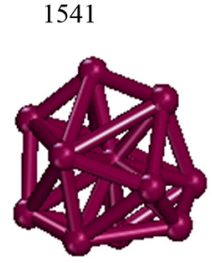

defected icosahedra

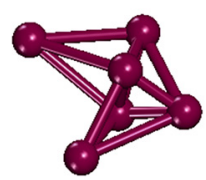

1431

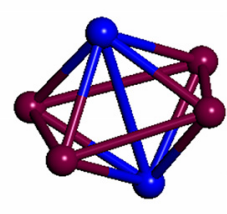

1441
Figure 4. Three-dimensional structures for some typical bonded pairs.

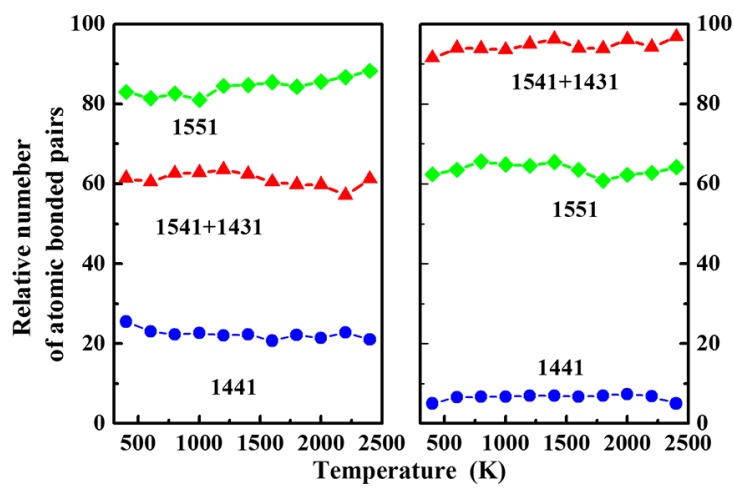

Figure 5. Relative number of various bonded pairs with the temperature. Left: 1.4 GPa. Right: $4 \mathrm{GPa}$.

an icosahedron, two of the 1551 pairs are transformed into the 1541 pairs, and two are transformed in the 1431 pairs. This trend represents a type of a defected icosahedral order. Through our simulation, the ratios of the four types of pairs do not change sensitively as the temperature decreases at 4GPa. A striking feature is that at the pressure of $4 \mathrm{GPa}$, the number of 1441 pairs corresponding to the $b c c$ crystalline structure reaches a very low percentage, as shown in Figure 5.

\section{References}

1. Cheng YQ, Ma E and Sheng HW. Atomic level structure in multicomponent bulk metallic glass. Physical Review Letters. 2009; 102(24):245501. http://dx.doi.org/10.1103/ PhysRevLett.102.245501. PMid:19659024.

2. Wang WH, Okada T, Wen P, Wang XL, Pan MX, Zhao $\mathrm{DQ}$, et al. Effect of pressure on nucleation and growth in the $\mathrm{Zr}_{46.75} \mathrm{Ti}_{8.25} \mathrm{Cu}_{7.5} \mathrm{Ni}_{10} \mathrm{Be}_{27.5}$ bulk glass-forming alloy investigated using in situ X-ray diffraction. Physical Review B: Condensed Matter and Materials Physics. 2003; 68(18):184105. http:// dx.doi.org/10.1103/PhysRevB.68.184105.

3. Dubrovinsky L, Dubrovinskaia N, Narygina O, Kantor I, Kuznetzov A, Prakapenka VB, et al. Body-centered cubic IronNickel alloy in earth's core. Science. 2007; 316(5833):1880-1883. http://dx.doi.org/10.1126/science.1142105. PMid:17600212.

4. Belonoshko AB, Ahuja R and Johansson B. Stability of the body-centred-cubic phase of iron in the Earth's inner core.
At this pressure, the number of the 1551 pairs following those of the 1541 and 1431 pairs, but it becomes the leading type of pairs at $1.4 \mathrm{GPa}$. Besides, it should be noted that, at $1.4 \mathrm{GPa}$, there is a distinct improvement of the number of 1441 pairs. Since the clusters with 1551 pairs have a five-fold symmetry, the number of such pairs is a direct measurement of the degree of the icosahedral order in the alloy. In conclusion, Figure 5 demonstrates the number of the full icosahedra cluster is at the second place, following those of the defected icosahedra cluster at $4 \mathrm{GPa}$, it becomes the leading type of pairs at $1.4 \mathrm{GPa}$. Moreover, not only the number of full icosahedra clusters but also the number of $b c c$ crystalline structures (1441 pairs) increased. It is the reason of the formation of the unusual feature of RDF (Figure 2) and projection (Figure 3) which is named as "intermediate structure". This intermediate structure is composed of full icocahedral and $b c c$ structure. During the cooling process of the system melt, there exists a competition of the local structures among the icocahedral structure, defected structures, $b c c$ structure (the crystal structure of this metal), etc. In the present case, more defect icosahedra are obtained under HP. At the pressure of $1.4 \mathrm{GPa}$, the structure composing of full icocahedral and $b c c$ are obtained in the system.

\section{Conclusion}

Our simulation reveals several important effects of high pressure on the structure evolution of Fe through the fast cooling process. At pressure of $1.4 \mathrm{GPa}$, the system passes from $b c c$ structure to a less closed packed structure which composed of full icosahedra clusters and $b c c$ structure. With the increase of pressures, an amorphous state was observed, and a compacted local structure with more defected icosahedra is obtained.

\section{Acknowledgements}

This work was supported by the National Basic Research Program of China (2013CB733000) and the National Natural Science Foundation of China (51271162, 51271161).

Nature. 2003; 424(6952):1032-1034. http://dx.doi.org/10.1038/ nature01954. PMid:12944963.

5. Vočadlo L, Alfè D, Gillan MJ, Wood IG, Brodholt JP and Price GD. Possible thermal and chemical stabilization of body-centredcubic iron in the Earth's core. Nature. 2003; 424(6948):536-539. http://dx.doi.org/10.1038/nature01829. PMid:12891353.

6. Lin JF, Heinz DL, Campbell AJ, Devine JN and Shen G. Ironsilicon alloy in Earth's core? Science. 2002; 295(5553):313-315. http://dx.doi.org/10.1126/science.1066932. PMid:11786640.

7. Lee KY and Ray JR. Mechanism of pressure-induced martensitic phase transformations: a molecular-dynamics study. Physical Review B: Condensed Matter and Materials Physics. 1989; 39(1):565-574. http://dx.doi.org/10.1103/PhysRevB.39.565. PMid:9947189.

8. Morishita T. How does tetrahedral structure grow in liquid Silicon upon supercooling. Physical Review Letters. 2006; 
97(16):165502. http://dx.doi.org/10.1103/PhysRevLett.97.165502. PMid: 17155410 .

9. Naumis GG. Variation of the glass transition temperature with rigidity and chemical composition. Physical Review B: Condensed Matter and Materials Physics. 2006; 73(17):172202. http://dx.doi.org/10.1103/PhysRevB.73.172202.

10. Mezger M, Reichert H, Ramsteiner IB, Udyansky A, Shchyglo $\mathrm{O}$, Bugaev VN, et al. Temperature and concentration dependence of the effective pair interaction parameters in Ni-Pd from highenergy X-ray diffuse scattering. Physical Review B: Condensed Matter and Materials Physics. 2006; 73(18):184206. http:// dx.doi.org/10.1103/PhysRevB.73.184206.

11. Hemley RJ and Mao HK. In situ studies of Iron under pressure: new windows on the earth's core. International Geology Review. 2001; 43(1):1-30.

12. Sutton AP and Chen J. Long-range finnis-sinclair potentials. Philosophical Magazine Letters. 1990; 61(3):139-146. http:// dx.doi.org/10.1080/09500839008206493.

13. Daw MS and Baskes MI. Embedded-atom method: derivation and application to impurities, surfaces, and other defects in metals. Physical Review B: Condensed Matter and Materials Physics. 1984; 29(12):6443-6453. http://dx.doi.org/10.1103/ PhysRevB.29.6443.

14. Srolovitz D, Vitek V and Egami T. An atomistic study of deformation of amorphous metals. Acta Metallurgica. 1983; 31(2):335-352. http://dx.doi.org/10.1016/0001-6160(83)90110-4.

15. Finnis MW and Sinclair JE. A simple empirical N-body potential for transition metals. Philosophical Magazine A. 1984; 50(1):4555. http://dx.doi.org/10.1080/01418618408244210.

16. Verlet L. Computer "experiments" on classical fluids. I. Thermodynamical properties of Lennard-Jones molecules. Physical Review. 1967; 159(1):98-103. http://dx.doi.org/10.1103/ PhysRev.159.98.

17. Andersen HC. Molecular dynamics simulations at constant pressure and/or temperature. The Journal of Chemical Physics. 1980; 72(4):2384. http://dx.doi.org/10.1063/1.439486.

18. Honeycutt JD and Andersen HC. Molecular dynamics study of melting and freezing of small Lennard-Jones clusters. Journal of Physical Chemistry. 1987; 91(19):4950-4963. http://dx.doi. org/10.1021/j100303a014. 